\title{
Superrotation of Earth's Inner Core, Extraterrestrial Impacts, and the Effective Viscosity of Outer Core
}

\author{
Pirooz Mohazzabi ${ }^{1}$ and John D. Skalbeck ${ }^{2}$ \\ ${ }^{1}$ Department of Mathematics and Physics, University of Wisconsin-Parkside, Kenosha, WI 53141, USA \\ ${ }^{2}$ Department of Geosciences, University of Wisconsin-Parkside, Kenosha, WI 53141, USA \\ Correspondence should be addressed to Pirooz Mohazzabi; mohazzab@uwp.edu
}

Received 13 October 2014; Revised 12 March 2015; Accepted 6 April 2015

Academic Editor: Petr Vaníček

Copyright (c) 2015 P. Mohazzabi and J. D. Skalbeck. This is an open access article distributed under the Creative Commons Attribution License, which permits unrestricted use, distribution, and reproduction in any medium, provided the original work is properly cited.

\begin{abstract}
The recently verified superrotation of Earth's inner core is examined and a new model is presented which is based on the tidal despinning of the mantle and the viscosity of the outer core. The model also takes into account other damping mechanisms arising from the inner core superrotation such as magnetic and gravitational coupling as well as contribution from eddy viscosity in the outer core. The effective viscosity obtained in this model confirms a previously well constrained value of about $10^{3} \mathrm{~Pa}$ s. In addition, the model shows that the currently measured superrotation of the inner core must be almost exactly equal to its asymptotic or steady-state value. The effect of extraterrestrial impacts is also investigated, and it is shown that perturbations due to such impacts can only persist over a short geological time.
\end{abstract}

\section{Introduction}

In 1996, Song and Richards analyzed two types of seismic waves traveling through Earth's solid and fluid cores, respectively [1]. They discovered a differential travel time between the two waves, which increased systematically by about $0.3 \mathrm{~s}$ from the year 1967 to the year 1995. This temporal change was later attributed to a shift of the lateral velocity gradient in the inner core caused by the inner core rotation $[2,3]$. Subsequent studies provided further support for the faster rotation of Earth's solid inner core compared to the rest of the planet [4-7].

Because of the anisotropy due to wood-like grain structure of the crystalline iron in the inner core, the speed of seismic waves is different in different directions [8]. If the inner core rotates faster than the rest of Earth, seismic waves that are generated at the same place on Earth but decades apart will have different travel times passing through the inner core and detected diametrically across the globe. Accurate recent investigations, using high-quality waveform doublets generated in the South Sandwich Islands region and detected in and near Alaska, have revealed definite temporal changes over a period of up to 35 years [9]. These experiments confirm that Earth's inner core rotates faster than the mantle and crust (hereafter simply referred to as mantle), with a superrotation of about 0.27 to $0.53 \mathrm{deg} / \mathrm{yr}$.

Estimates of the inner core differential rotation rates range from low values of 0.2 to $0.3 \mathrm{deg} / \mathrm{yr}$ [2] to high values of $3 \mathrm{deg} / \mathrm{yr}$ [10]. Deuss [11] presents a compilation of studies on inner core differential rotation rates as a function of publication year that suggests more recent data supports lower inner core differential rotation rates; however, the data is quite scattered. The author cites a recent study [12] that reports no inner core rotation and another study [13] that shows subrotation but the majority of the studies report inner core superrotation with respect to the mantle.

Several Earth-based mechanisms have been suggested for Earth's inner-core differential rotation. Gubbins [14] attributes the phenomenon to the existence of a large toroidal magnetic field inside Earth's core which, together with the dipole components, produces a magnetic torque on the inner core that tends to accelerate it relative to the mantle. 
He then assumes that some equilibrium is reached so that the inner core rotates with constant angular velocity and experiences zero net torque. Glatzmaier and Roberts [15] numerically solve the self-consistent magnetohydrodynamic equations that describe thermal convection and magnetic field generation in a rapidly rotating spherical fluid shell with a solid conducting inner core. Their solution, which serves as an analog for the geodynamo, shows that viscous and magnetic coupling of the outer core with the inner core and the mantle causes time-dependent variations in their respective rotation rates; the inner core usually rotates faster than the mantle. Buffett and Glatzmaier [16] allowed gravitational coupling between the inner core and the mantle by incorporating viscous deformation of the inner core into their numerical simulations of the geodynamo. They discovered that differential rotation between the inner core and the mantle is permitted by allowing the inner core to deform. Numerical calculations by Aurnou et al. [17] showed excess temperature inside the tangent cylinder surrounding the inner core which generates a prograde thermal wind and a strong azimuthal magnetic field inside the tangent cylinder. They conclude that the electromagnetic torque on the inner core resulting from induced azimuthal magnetic fields and the ambient poloidal field equilibrate when certain conditions are met. Dumberry [18] studied the steady and time-dependent rates of inner core rotation based on angular momentum balance between the inner core, fluid core, and mantle. He concluded that the rotational rate of an oscillating inner core is constrained by the changes in mantle rotation induced by gravitational coupling.

Tkalčić and others [19] analyzed earthquake doubles to construct a model for inner core differential rotation rates of 0.25 to $0.48 \mathrm{deg} / \mathrm{yr}$ with decadal fluctuations around the mean of $1 \mathrm{deg} / \mathrm{yr}$. The authors suggest that these decadal fluctuations can account for discrepancies between previous core rotation models and agree with recent geodynamo simulations. A three-dimensional model by Livermore and others [20] suggests that axial electromagnetic torque is the dominant influence for inner core differential rotation and that decadal variations of the magnetic field may drive the quasioscillatory nature of the inner core differential rotation.

Magnetic coupling between the inner and outer cores seems to play a role not only in the superrotation of the inner core but also in the generation of the Earth's magnetic field. Su et al. [10] reported an anomalous variation in the inner core orientation that temporally coincided with the geomagnetic "jerk" (a sudden change in the strength of Earth's magnetic field of 1969-1970). This suggests a correlation between Earth's magnetic field and the inner core superrotation. Glatzmaier and Roberts [21] have suggested that the inner core rotates in response to the magnetic torque $\Gamma_{B}$ and the viscous torque $\Gamma_{v}$ to which it is subjected, with $\Gamma_{B}+\Gamma_{v}=0$. The magnetic torque drags the inner core eastward and the viscous torque acts westward. Nevertheless, they state that even though the fluid viscosity in their model is several orders of magnitude greater than is likely for real Earth, the viscous torque on the inner core has little effect.

Although each of these theories provides a reasonable explanation for the differential rotation of the inner core, there is no experimental evidence supporting one over the others, and they are all based on processes that are assumed to be taking place inside Earth. Furthermore, about a decade before the most recent data of 0.27 to $0.53 \mathrm{deg} / \mathrm{yr}$ were published, calculations based on magnetic coupling between the inner and outer cores suggested superrotations that were about an order of magnitude higher than these values [10, 21].

Perhaps the most significant and tangible external factor responsible for the inner core superrotation is the tidal forces $[22,23]$. Dissipation of tidal energy in oceans and transfer of angular momentum between Earth, Moon, and the Sun results in torques on the mantle causing it to dispin [24], which gradually increases the length of the day continuously. This despinning of the mantle leaves the inner core with a small excess eastward rotational velocity relative to the mantle. Su et al. [10] used the known tidal increase in the length of day of approximately $2 \mathrm{~ms}$ per century to extrapolate backward and conclude that the inner core was rotating with the same period as the mantle about $10^{5}$ years ago. The recent confirmation of superrotation of Earth's inner core by Zhang and others [9] prompted us to examine this phenomenon in the context of tidal effects from a phenomenological point of view.

In addition to the oceanic tidal effects, yet another external factor could contribute to the differential rotation of the Earth's inner core which has not been addressed in the literature, Earth impacts. Since the formation of our Solar System some 4.6 billion years ago, collisions and impacts have played a fundamental role in establishing its characteristics, ranging from the accretion of planetesimals and the early formation of planets $[25,26]$ to the recent series of impacts on Jupiter by the fragments of Shoemaker-Levy 9 comet in July 1994 [27]. These collisions and impacts have affected the dynamics of various components of the Solar System. For example, most planets have obliquity or axial tilt with respect to their orbital planes about the Sun. Earth has an obliquity of about $23.5^{\circ}$ while Uranus, the third largest planet in the Solar System, has an obliquity of about $97^{\circ}$. In other words, Uranus is tilted on its side so that its rotation axis is nearly in its orbital plane about the Sun. Yet, giant planets are believed to form with nearly zero obliquity [28]. The axial tilts of planets are believed to have been caused by major impacts $[29,30]$.

The above discussion is the motivation for examining a second question in this paper. Because Earth's solid inner core rotates inside the fluid outer core, is it possible for bolide impacts to alter the angular velocity of Earth's mantle relative to the inner core, resulting in a superrotation, a subrotation or a transition from one to the other? If so, is it possible for these impacts to result in differential rotations that are comparable to the experimentally observed superrotation values, and how long would it take for such perturbations to damp out?

The model presented here could equally be applied for conditions that would generate subrotation or no differential rotation depending on the size and angle of bolide impact. Examination of the probability of direction of differential rotation is beyond the scope of this paper. The focus here is to examine the potential for bolide impact contribution if superrotation of the inner core is present. 


\section{Tidal Despinning of the Mantle and Viscosity of the Outer Core}

As stated earlier, dissipation of tidal energy in the oceans results in gradual despinning of the mantle, which leaves the inner core with a small excess relative eastward differential rotation relative to the mantle called superrotation [24]. This differential rotation is communicated between the mantle and the inner core though a Couette flow in the fluid outer core which tends to damp the superrotation.

Consider two concentric solid spheres separated by a fluid layer between them with a coefficient of viscosity $\eta$. Let the radius of the inner sphere be $r_{1}$ and the inside radius of the outer sphere be $r_{2}$. Then, if the inner sphere rotates with an angular velocity $\omega$ with respect to the outer sphere, the damping torque $\Gamma_{\eta}$ on it due to the fluid viscosity is given by [31]

$$
\Gamma_{\eta}=8 \pi \eta\left(\frac{r_{1}^{3} r_{2}^{3}}{r_{2}^{3}-r_{1}^{3}}\right) \omega .
$$

Although Dai and others [32] have presented seismic reflection data suggesting significant topography on the inner core boundary, this model can be applied to Earth's inner core and mantle, with the fluid between them being the outer core. Here the effect of the topography is absorbed in the estimate of effective viscosity.

Using (1), the rotational equation of motion of the inner core,

$$
\Gamma_{\text {net }}=I \frac{d \omega}{d t}
$$

becomes

$$
I \alpha-8 \pi \eta\left(\frac{r_{1}^{3} r_{2}^{3}}{r_{2}^{3}-r_{1}^{3}}\right) \omega=I \frac{d \omega}{d t},
$$

where $I$ is the rotational inertia of the solid inner sphere. The left hand side in this equation is the net torque on the inner sphere. The first term represents the torque due to the tidal effects, which tends to increase the eastward rotational velocity $\omega$ of the inner core relative to the mantle. $\alpha$ is the constant rate at which the rotational speed of the mantle slows down due to the tidal effects. It has a value of 2.3 milliseconds per day per century [33] or $3.45 \times 10^{-5} \mathrm{deg} / \mathrm{yr}^{2}$. The second term on the left hand side of (3) is the viscous damping torque.

Writing $I$ in terms of the inner core density $\rho_{1}$ and the inner core radius $r_{1}$, reduces (3) to

$$
\frac{d \omega}{d t}=-\frac{15 \eta}{\rho_{1} r_{1}^{2}}\left(\frac{r_{2}^{3}}{r_{2}^{3}-r_{1}^{3}}\right) \omega+\alpha .
$$

Furthermore, defining the characteristic time $\tau$ by

$$
\tau=\frac{\rho_{1} r_{1}^{2}}{15 \eta}\left[1-\left(\frac{r_{1}}{r_{2}}\right)^{3}\right]
$$

reduces (4) to

$$
\frac{d \omega}{d t}=-\frac{\omega}{\tau}+\alpha
$$

Integration of this equation using the initial condition $\omega(0)=$ 0 gives

$$
\omega=\alpha \tau\left(1-e^{-t / \tau}\right)
$$

From this equation, we see that $\tau$ is in fact the relaxation time for the process. With the value of $\tau$ obtained from (5), this equation gives the superrotation of the inner core $\omega$ at any time $t$ after the dissipation of tidal energy began, about $4 \times$ $10^{9}$ years ago, since oceans existed as early as the relatively stable Earth. The value of $\alpha$, as mentioned earlier, is $3.45 \times$ $10^{-5} \mathrm{deg} / \mathrm{yr}^{2}$.

Earth's inner core has a radius of $1.220 \times 10^{6} \mathrm{~m}$ and a density of $13000 \mathrm{~kg} \mathrm{~m}^{-3}$. The liquid outer core has a radius of $3.473 \times 10^{6} \mathrm{~m}$ [34]. The viscosity of the outer core, however, is highly uncertain depending on its method of determination. In fact, its estimated values from various sources span over 14 orders of magnitude from $10^{-3}$ to $10^{11} \mathrm{~Pa} \mathrm{~s}$ [35]. For example, de Wijs et al. [36] report a value of $1.5 \times 10^{-2} \mathrm{Pas}$ with an uncertainty of a factor of three through dynamical first principles simulations of liquid iron. Similarly, Rutter et al. [37] report a value of $1.6 \times 10^{-2} \mathrm{~Pa}$ s using experimental high pressure study of liquid $\mathrm{Fe}-\mathrm{S}$ system. On the other hand, using amplitude of forced nutation, Molodenskiy [38] finds a value of about $1 \times 10^{6} \mathrm{~Pa} \mathrm{~s}$.

Although the viscosity of the Earth's outer core is notoriously uncertain, it is plausibly considered to be bounded by $10^{2} \mathrm{Pas} \leq \eta \leq 10^{11} \mathrm{Pas}[32,34]$. Estimates of the fluid outer cores viscosity show variation from $10^{2} \mathrm{~Pa}$ at the top of the outer core to $10^{11} \mathrm{Pas}$ at the bottom near the inner core boundary using Arrhenius extrapolation of pressure dependencies for laboratory measurements on liquid iron $[39,40]$. A viscosity value of $1.22 \times 10^{11} \mathrm{~Pa}$ s near the solid inner core was found by Smylie [41] using Ekman layer theory to estimate viscous drag forces from Coriolis splitting of the two equatorial translational models of oscillation of the solid inner core. Palmer and Smylie [42] found a viscosity value of $615 \mathrm{~Pa}$ s near the top of the outer core from the free decay of free core nutations. Smylie et al. [43] present a viscosity estimate of $2.371 \times 10^{3} \mathrm{~Pa}$ for the top of the outer core which relies on the decay of free core nutations method and an estimate for the bottom of the outer core of $1.247 \times 10^{11} \mathrm{~Pa} \mathrm{~s}$ from the method of Smylie [41] and accounting for pressure dependence of the activation volume. The authors found the viscosity profile fits a nearly log-linear trend across the outer core but values still vary by 9 orders of magnitude. Smylie [44] presents this log-linear viscosity profile for the outer core based on the Arrhenius description of temperature and pressure of the viscosity by Brazhkin [39] and also reports a mean viscosity value of $3124 \mathrm{~Pa}$ s at the top of the outer core.

Therefore, instead of using a highly variable value of the viscosity to calculate the superrotation of the inner core, we solve the inverse problem. We use the measured value of the superrotation $\omega$ to find the viscosity of the outer core. To do so, we first solve the transcendental equation (7) numerically for the relaxation time $\tau$, using $\omega=0.4 \mathrm{deg} / \mathrm{yr}$ (mean value of the measured superrotation), $\alpha=3.45 \times 10^{-5} \mathrm{deg} / \mathrm{yr}^{2}$, and $t=4 \times 10^{9} \mathrm{yr}$ (age of oceans). This gives a value $\tau=11600 \mathrm{yr}$ 
(or $3.66 \times 10^{11} \mathrm{~s}$ ). Then, we use (5) and solve for the viscosity of the outer core $\eta$. We find $\eta=3.37 \times 10^{3} \mathrm{~Pa}$. This number is in incredible agreement with the value obtained by Bills [24] and by Smylie [44]. Using a well described different model, Bills [24] reports an outer core viscosity of the order of $10^{3} \mathrm{~Pa}$ s, assuming a value of superrotation of about $1 \mathrm{deg} / \mathrm{yr}$. It is interesting to note that if we use $\omega=1 \mathrm{deg} / \mathrm{yr}$ (instead of $0.4 \mathrm{deg} / \mathrm{yr}$ ) in our calculations, we find $\eta=1.35 \times 10^{3} \mathrm{~Pa}$. Our calculated viscosity does not agree with the study by $\mathrm{Su}$ et al. [10] that implied a viscosity of $10^{-4} \mathrm{~Pa}$ s by ignoring electromagnetic forces and assuming differential rotation of $3 \mathrm{deg} / \mathrm{yr}$.

Admittedly, our values of the viscosity are calculated at the inner core boundary as our theoretical model considers rotation of the inner core. However, the extremely high viscosity of $10^{11} \mathrm{~Pa}$ near the inner core strongly couples the lower zone of the outer core to the inner core. Thus, the differential rotation effectively takes place further out in the outer core where the viscosity is considerably lower. Since we are solving the inverse problem, our calculated viscosity corresponds to this region where the differential rotation effectively takes place. Ironically, due to the functional form of (5), the values of the viscosity calculated for a superrotation of $0.4 \mathrm{deg} / \mathrm{yr}$ with $\rho_{1}=12000-13000 \mathrm{~kg} / \mathrm{m}^{3}$ and with $r_{1}$ ranging from the inner core radius all the way to $98 \%$ of the outer core radius vary in a narrow range of $10^{3}-10^{4} \mathrm{~Pa}$.

Let us now return to the notorious uncertainty in the reported values of outer core viscosity. Generally these numbers fall into two distinct categories. The high values are based on seismologic, geodetic, and geomagnetic observations of Earth $[32,34,41]$, whereas the low values are based on theory and laboratory investigations of liquid metals $[24,45,46]$. It has been suggested that this dichotomy is possibly due to contribution of eddy viscosity caused by fluid motion, whereas the liquid metal investigations only account for intrinsic or molecular viscosity [24]. In addition, it is possible that electromagnetic and gravitational couplings also play roles. Since all these effects arise from the differential rotation of the inner core; therefore, they should each be a function of $\omega$. Furthermore, according to Lenz' Law and LeChatelier Principle, they should all act in such a way to reduce or eliminate the cause, which is the inner core superrotation.

Let the combined torque resulting from all these damping effects, which for the sake of simplicity from now on we refer to as core coupling, be $\Gamma_{c}=\Gamma_{c}(\omega)$. Expanding this function in a Taylor series, we have

$$
\Gamma_{c}(\omega)=\Gamma_{c}(0)+\Gamma_{c}^{\prime}(0) \omega+\frac{1}{2} \Gamma_{c}^{\prime \prime}(0) \omega^{2}+\cdots .
$$

Because $\omega$ is small, we can neglect the second- and higherorder terms in the expansion. In addition, since we must have $\Gamma_{c}(0)=0$, we obtain

$$
\Gamma_{c}(\omega)=\Gamma_{c}^{\prime}(0) \omega .
$$

Adding this retarding torque to the left hand side of (3), we obtain

$$
I \alpha-8 \pi \eta\left(\frac{r_{1}^{3} r_{2}^{3}}{r_{2}^{3}-r_{1}^{3}}\right) \omega-\Gamma_{c}^{\prime}(0) \omega=I \frac{d \omega}{d t} .
$$

Repeating the calculations as before, instead of (6) we get

$$
\frac{d \omega}{d t}=-\left(\frac{1}{\tau}+\frac{1}{\mu}\right) \omega+\alpha,
$$

where $\mu$ is a constant with the dimension of time. Integration of this equation with the initial condition $\omega(0)=0$ gives

$$
\omega=\alpha \kappa\left(1-e^{-t / \kappa}\right),
$$

where $\kappa$ is defined by

$$
\frac{1}{\kappa}=\frac{1}{\tau}+\frac{1}{\mu}
$$

which is the new relaxation time, taking into account both the viscosity of the outer core as well as all other damping effects which we called core coupling.

Equation (12) has exactly the same functional form as (7) except that $\tau$ is replaced by $\kappa$. Therefore, if we solve this equation for $\kappa$ using the known value of $\omega$, we find the same value for $\kappa$ that we found for $\tau$ before; that is, $\kappa=11600 \mathrm{yr}$. Thus, the relaxation time of $11600 \mathrm{yr}$ corresponds not only to the viscosity of the outer core, but also to all other damping effects as well. Using this value in (5) and solving for $\eta$; therefore, gives an effective value for the outer core viscosity that could be greater than the actual value. It is interesting to note that since the relaxation time $\kappa$ is $11600 \mathrm{yr}$, the value of $\omega$ today is so close to its saturation value $\alpha \kappa$ that it would be impossible to measure the difference.

\section{Extraterrestrial Impacts}

Because geomagnetism and its reversals are associated with the differential rotation of Earth's inner core, therefore, any changes in the Earth's magnetic field can be attributed to a change in the inner core's superrotation. Muller and Morris [47] have suggested that the impact of a large extraterrestrial object on Earth can produce a geomagnetic reversal through a mechanism involving a sequence of events. If so, it would be possible for such an impact to alter the superrotation of the inner core. In what follows, we study the dynamics of a bolide impact and calculate the change in the steady-state value of $\alpha \kappa$ of the differential rotation of Earth's inner core resulting from such impacts. We then investigate the time scale over which such a perturbation in the inner core superrotation would damp out.

Consider an asteroid or comet of mass $m$ and velocity $\mathbf{v}$ relative to the center of Earth just before impact, as shown in Figure 1. For simplicity, we assume that the velocity vector of the asteroid or comet is in the equatorial plane of Earth. The center of mass of the bolide-Earth system is located at a distance of

$$
d_{\mathrm{cm}}=\frac{m R}{m+M}<\frac{m}{M} R
$$

from Earth's center, where $M$ is the mass of Earth and $R$ is its radius. For a typical bolide, $m \ll M$ and therefore $d_{\mathrm{cm}}$ is very 


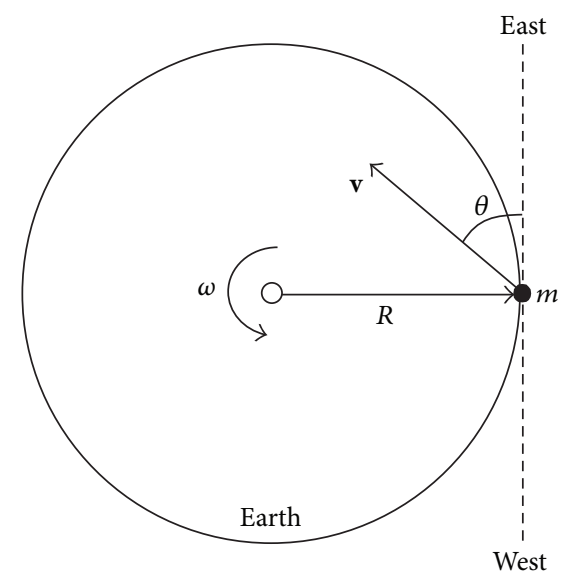

Figure 1: A bolide of mass $m$ and velocity $\mathbf{v}$ relative to Earth's center impacts Earth with an angle $\theta$ from the eastward direction. The velocity vector of the bolide just before the impact lies in the equatorial plane.

small. For example, for a bolide of radius $r=20 \mathrm{~km}$ and a density comparable to that of Earth, we have

$$
\frac{m}{M}=\left(\frac{r}{R}\right)^{3}=\left(\frac{20000}{6.378 \times 10^{6}}\right)^{3}=3.08 \times 10^{-8} .
$$

Then, with $R=6.378 \times 10^{6} \mathrm{~m}$, we find $d_{\mathrm{cm}}<0.2 \mathrm{~m}$, which is less than $20 \mathrm{~cm}$ from Earth's center. Therefore, we can safely take the center of mass of the bolide-Earth system at the moment of impact to be simply Earth's center.

The time rate of change of the total angular momentum of a system of particles is equal to the net external torque on the system, all measured with respect to some inertial coordinate system:

$$
\frac{d \vec{L}}{d t}=\vec{\Gamma}_{\text {net }}
$$

However, if (16) is written relative to the center of mass of the system, then it is valid even if the center of mass is accelerating [48].

For the bolide-Earth system, the net external torque about Earth's center is zero and the angular momentum of the system about that point is conserved during the impact. Therefore, we have

$$
I \Omega+m v R \cos \theta=(I+d I)(\Omega+d \Omega),
$$

where $v$ is the speed (magnitude of the velocity vector) of the bolide and $\theta$ is the angle between the bolide's velocity vector and the tangent to Earth in the direction of Earth's rotation, as shown in Figure 1. The first term on the left hand side is the angular momentum of Earth before the impact, in which $I$ is the rotational inertia of Earth and $\Omega$ is its angular velocity. The second term is the angular momentum of the bolide before the impact. The term on the right is the angular momentum of the combination after the impact, in which $d I$ and $d \Omega$ are very small compared to $I$ and $\Omega$, respectively.
Expanding the right hand side of (17), neglecting the second-order infinitesimal term $d I d \Omega$, and rearranging the remaining terms, we find

$$
m v R \cos \theta=I d \Omega+\Omega d I
$$

which may also be written as

$$
d(I \Omega)=m v R \cos \theta .
$$

Equation (19) simply states that the change of the angular momentum of Earth is that imparted to it by the bolide.

Dividing both sides of (18) $I \Omega$, we obtain

$$
\frac{d \Omega}{\Omega}=\frac{m v R \cos \theta}{I \Omega}-\frac{d I}{I} .
$$

Now, $d I=m R^{2}$ because the bolide lodges itself near Earth's surface. Furthermore, assuming Earth to be a solid sphere of uniform density for the moment (we address this point later on), we have $I=(2 / 5) M R^{2}$. Substituting in (20), we obtain

$$
\frac{d \Omega}{\Omega}=\frac{5}{2} \frac{m}{M}\left(\frac{v \cos \theta}{R \Omega}-1\right) .
$$

Finally, using

$$
\frac{m}{M}=\frac{\rho_{b}}{\rho}\left(\frac{r}{R}\right)^{3}
$$

where $\rho_{b}$ and $\rho$ are the mean densities of the bolide and Earth, respectively, we obtain

$$
\frac{d \Omega}{\Omega}=\frac{5}{2} \frac{\rho_{b}}{\rho}\left(\frac{r}{R}\right)^{3}\left(\frac{v \cos \theta}{R \Omega}-1\right) .
$$

Equation (23) measures the fractional change in Earth's angular velocity as a result of a bolide impact. This equation assumes that the velocity vector of the bolide is in the equatorial plane of Earth, and that Earth is a solid sphere of uniform density. However, since the outer core is fluid, it does not immediately transfer the angular momentum resulting from the bolide impact to the solid inner core. Therefore, we need to only consider the rotation of the mantle in our calculations. The moment of inertia of the mantle, $I_{m}$, is the difference between the moment of inertia of the entire Earth and that of the inner and outer cores:

$$
\begin{aligned}
I_{m} & =I-I_{c}=\left(1-\frac{I_{c}}{I}\right) I=\left(1-\frac{M_{c} R_{c}^{2}}{M R^{2}}\right) I \\
& =\left[1-\left(\frac{\rho_{c}}{\rho}\right)\left(\frac{R_{c}}{R}\right)^{5}\right] I,
\end{aligned}
$$

where $R_{c}$ is the radius of the outer core, $\rho_{c}$ is the combined mean density of the inner and outer cores, and $\rho$ is the mean density of the entire Earth. If (24) is written as

$$
I_{m}=\gamma I,
$$


where

$$
\gamma=1-\left(\frac{\rho_{c}}{\rho}\right)\left(\frac{R_{c}}{R}\right)^{5}
$$

(23) becomes

$$
\frac{d \Omega}{\Omega}=\frac{5}{2 \gamma} \frac{\rho_{b}}{\rho}\left(\frac{r}{R}\right)^{3}\left(\frac{v \cos \theta}{R \Omega}-1\right),
$$

where now $\Omega$ and $d \Omega$ refer, respectively, to the angular velocity and the change in angular velocity of the mantle.

Because Earth's mass and mean radius are, respectively, $M=5.976 \times 10^{24} \mathrm{~kg}$ and $R=6.371 \times 10^{6} \mathrm{~m}$, we calculate a mean density of $\rho=5517 \mathrm{~kg} \mathrm{~m}^{-3}$ for Earth. Furthermore, the radius of the outer core and the combined mean density of the inner and outer cores are, respectively, $R_{c}=3.473 \times 10^{6} \mathrm{~m}$ and $\rho_{c}=11770 \mathrm{~kg} \mathrm{~m}^{-3}$ [34]. These data, together with (26), give $\gamma=0.8973$. Thus, the correction to the fractional change in the angular velocity of the mantle as compared to entire Earth is only about $10.3 \%$.

The change in the angular velocity of the mantle as a result of asteroid or comet impact can be positive, negative, or zero depending on the parameters involved in (27). There are tens of thousands of asteroids in the Solar System and about 220 have diameters greater than $100 \mathrm{~km}$. Ceres, the largest known asteroid, has a diameter of about $950 \mathrm{~km}$ [49]. The orbit of some of these asteroids is such that they could potentially be perturbed into a collision course with Earth.

An object attracted by Earth's gravitational field from large distances will impact Earth with a minimum speed of about $11 \mathrm{~km} \mathrm{~s}^{-1}$. A more likely collision, however, is when Earth intercepts an object that is being attracted by the Sun which has a much more powerful gravitational attraction and the impact speed will be much higher. In fact, it has been estimated that if the bolide responsible for the Chicxulub crater was a comet, it must have struck Earth with a speed as high as 70 or $80 \mathrm{~km} \mathrm{~s}^{-1}$ [50].

To obtain a numerical estimate for the change of the angular velocity of the mantle as a result of an asteroid or comet impact, we consider a bolide of radius $15 \mathrm{~km}$ and a density comparable to that of Earth, impacting Earth with a speed of $40 \mathrm{~km} \mathrm{~s}^{-1}$. The angle of impact $\theta$ can be anywhere from 0 to $180^{\circ}$ as shown in Figure 1. For $\theta$ between 0 and $90^{\circ}$, the impact speeds up the rotation of the mantle relative to the inner core, and for $\theta$ between $90^{\circ}$ and $180^{\circ}$ the impact slows it down. Let us consider the special cases of near glancing angles $\theta=0$ and $\theta=180^{\circ}$. Because Earth's angular velocity is

$$
\Omega=\frac{2 \pi}{24 \times 3600}=7.2722 \times 10^{-5} \mathrm{rad} / \mathrm{s}
$$

from (27), with $\theta=0$, we find $d \Omega=2.257 \times 10^{-10} \mathrm{rad} / \mathrm{s}=$ $0.41 \mathrm{deg} / \mathrm{yr}$. On the other hand, if the impact is in the direction $\theta=180^{\circ}$, we find a change in the mantle's angular velocity of $d \Omega=-0.42 \mathrm{deg} / \mathrm{yr}$. Because the change of the differential rotation of the inner core relative to the mantle as a result of an impact is $\delta \omega_{i}=-d \Omega$, the impact in the former case decreases the superrotation of the inner core by $0.41 \mathrm{deg} / \mathrm{yr}$

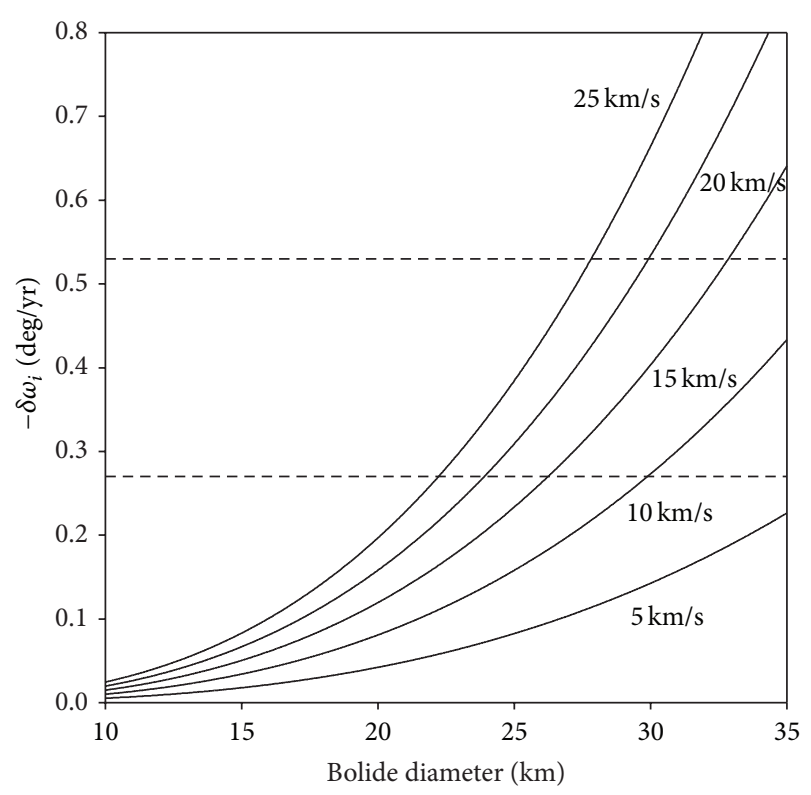

FIGURE 2: Magnitude of the impact-induced superrotation of the Earth's inner core $\left(-\delta \omega_{i}\right)$ as a function of bolide diameter and speed. The horizontal dashed lines indicate the range of superrotation reported by Zhang et al. [9].

and that in the latter case increases it by $0.42 \mathrm{deg} / \mathrm{yr}$. A strong enough impact in the direction $\theta=0$ can impart a change in $\omega$ that exceeds the value of $\alpha \kappa$, resulting in a subrotation of the inner core.

Figure 2 shows plots of the change in superrotation of Earth's inner core, $\delta \omega_{i}$, as a result of bolide impacts of various diameters and speeds. Bolide densities are assumed to be comparable to that of Earth and the impacts are in the direction $\theta=180^{\circ}$. The limits of the measured values of Earth's inner core superrotation are also shown in the same figure for comparison.

Immediately after impact, the value of the differential rotation of the inner core relative to the mantle changes to $\alpha \kappa+\delta \omega_{i}$. To find out how this new value decays, we solve (11) using the initial condition $\omega(0)=\alpha \kappa+\delta \omega_{i}$. This gives

$$
\omega=\alpha \kappa+\delta \omega_{i} e^{-t / \kappa}
$$

Because $\kappa=11600 \mathrm{yr}$, (29) shows that in a time scale of a few tens of thousand years the effect of bolide impact damps out. Therefore, impacts only contribute to geologically short-term perturbations in the steady-state value $\alpha \kappa$ of the inner-core superrotation.

\section{Discussion and Conclusion}

There are a number of existing theories explaining the superrotation of Earth's inner core. Most of these theories are based on internal Earth processes. Although each theory provides a reasonable account for the phenomenon, there is not sufficient experimental evidence to support one theory over the others. The external tidal effect, on the other hand, appears to be one of the most reasonable explanations, which 
is also supported by experimental evidence and measurements.

The frictional torques generated by the tidal effects tend to slow down the rotational motion of the mantle, thereby increasing the superrotation of the inner core. This effect is counterbalanced by the viscous forces of the Earth's fluid outer core as well as other effects such as the electromagnetic and gravitational couplings and eddy viscosity caused by fluid motion in the outer core. The combined effect gives rise to an effective viscosity of the outer core on the order of $10^{3} \mathrm{Pas}$, in agreement with a previously reported value based on a different model [24]. The viscosity of the molten material of the outer core without these additional contributions, however, could be considerably less. Because the relaxation time for the inner core superrotation is of the order of $10^{4} \mathrm{yr}$, the superrotation today is almost exactly equal to its saturated or steady-state value.

Bolide impacts, yet another external factor, are possibly responsible for sudden changes in the differential rotation of Earth's inner core from time to time and can render changes comparable in magnitude to the presently observed values. Although the existing geological evidence indicates few major bolide impacts with Earth in recent geological times, the large number of craters on the Moon strongly suggests that Earth, with a considerably larger cross-sectional area, has also been subject to a large number of impacts since its formation. Most of the resulting craters, however, have been hidden or destroyed by Earth's weathering and tectonic processes. Therefore, the number of bolide impacts on Earth is not limited to the number of existing craters but rather is much higher. Furthermore, as our science and technology advances, we discover more impact craters on Earth of which we were previously unaware. For example, it was originally believed that the Vredefort dome in South Africa was formed by a volcanic explosion. But in the mid-1990s, evidence revealed that it was in fact the site of a huge bolide impact about two billion years ago. Evidence of four impacts older than Vedefort that occurred between 3.2 to 3.5 billion years ago has also been found in South Africa. These sites, however, are not easily recognizable as impact structures on Earth's surface.

Asteroids approaching Earth and the Moon have an average speed of about $17 \mathrm{~km} \mathrm{~s}^{-1}$ and, depending on their composition, can have densities ranging in the extreme from about $3 \mathrm{~kg} \mathrm{~m}^{-3}$ (rock) to about $8 \mathrm{~kg} \mathrm{~m}^{-3}$ (iron). The diameters can be as high as tens of kilometers. As shown in Figure 2, there is a wide range of diameters and velocities for an impacter which can cause superrotations of Earth's inner core comparable to the observed values. In fact, several asteroids among the near Earth objects fall in this category. For example, 1036 Ganymed, the largest asteroid in this class is about $32 \mathrm{~km}$ across. According to Figure 2, with an impact speed of about $10 \mathrm{~km} \mathrm{~s}^{-1}$, an asteroid of this size can cause a superrotation in Earth's inner core that is in the range of the observed values. Furthermore, cumulative effects from smaller impacts may also result in such superrotations.

Nonetheless, as stated earlier, these impacts that can change the steady-state differential rotation of the Earth's inner core can be viewed as short lived perturbations in geological terms, with decaying times of the order of a few tens of thousands of years. The discrepancy between our damping time scale of a few tens of thousands of years and those obtained by Tkalčić et al. [19] and by Livermore et al. [20] of the order of decades is the result of invoking different mechanisms and models for the inner-core superrotation.

\section{Conflict of Interests}

The authors declare that there is no conflict of interests regarding the publication of this paper.

\section{References}

[1] X. D. Song and P. G. Richards, "Seismological evidence for differential rotation of the earth's inner core," Nature, vol. 382, no. 6588 , pp. 221-224, 1996.

[2] K. C. Creager, "Inner core rotation rate from small-scale heterogeneity and time-varying travel times," Science, vol. 278, no. 5341, pp. 1284-1288, 1997.

[3] X. D. Song, "Joint inversion for inner core rotation, inner core anisotropy, and mantle heterogeneity," Journal of Geophysical Research B: Solid Earth, vol. 105, no. 4, pp. 7931-7943, 2000.

[4] J. E. Vldale, D. A. Dodge, and P. S. Earle, "Slow differential rotation of the Earth's inner core indicated by temporal changes in scattering," Nature, vol. 405, no. 6785, pp. 445-448, 2000.

[5] X. D. Song, "Time dependence of PKP(BC)-PKP(DF) times: could this be an artifact of systematic earthquake mislocations?" Physics of the Earth and Planetary Interiors, vol. 122, no. 3-4, pp. 221-228, 2000.

[6] X. D. Song and A. Y. Li, "Support for differential inner core superrotation from earthquakes in Alaska recorded at South Pole station," Journal of Geophysical Research B: Solid Earth, vol. 105, no. 1, pp. 623-630, 2000.

[7] J. D. Collier and G. Helffrich, "Estimate of inner core rotation rate from United Kingdom regional seismic network data and consequences for inner core dynamical behaviour," Earth and Planetary Science Letters, vol. 193, no. 3-4, pp. 523-537, 2001.

[8] R. A. Kerr, "Earth's inner core is running a tad faster than the rest of the planet," Science, vol. 309, no. 5739, p. 1313, 2005.

[9] J. Zhang, X. Song, Y. Li, P. C. Richards, X. Sun, and F. Waldhauser, "Inner core differential motion confirmed by earthquake waveform doublets," Science, vol. 309, no. 5739, pp. 1357-1360, 2005.

[10] W.-J. Su, A. M. Dziewonski, and R. Jeanloz, "Planet within a planet: rotation of the inner core of earth," Science, vol. 274, no. 5294, pp. 1883-1887, 1996.

[11] A. Deuss, "Heterogeneity and anisotropy of earth's inner core," Annual Review of Earth and Planetary Sciences, vol. 42, no. 1, pp. 103-126, 2014.

[12] A. M. Mäkinen and A. Deuss, "Global seismic body-wave observations of temporal variations in the Earth's inner core, and implications for its differential rotation," Geophysical Journal International, vol. 187, no. 1, pp. 355-370, 2011.

[13] A. Souriau, "New seismological constraints on differential rotation of the inner core from Novaya Zemlya events recorded at DRV, Antarctica," Geophysical Journal International, vol. 134, no. 2, pp. F1-F5, 1998.

[14] D. Gubbins, "Rotation of the inner core," Journal of Geophysical Research, vol. 86, no. 12, pp. 11695-11699, 1981. 
[15] G. A. Glatzmaier and P. H. Roberts, "A three-dimensional convective dynamo solution with rotating and finitely conducting inner core and mantle," Physics of the Earth and Planetary Interiors, vol. 91, no. 1-3, pp. 63-75, 1995.

[16] B. A. Buffett and G. A. Glatzmaier, "Gravitational braking of inner-core rotation in geodynamo simulations," Geophysical Research Letters, vol. 27, no. 19, pp. 3125-3128, 2000.

[17] J. M. Aurnou, D. Brito, and P. L. Olson, "Mechanics of inner core super-rotation," Geophysical Research Letters, vol. 23, no. 23, pp. 3401-3404, 1996.

[18] M. Dumberry, "Geodynamic constraints on the steady and time-dependent inner core axial rotation," Geophysical Journal International, vol. 170, no. 2, pp. 886-895, 2007.

[19] H. Tkalčić, M. Young, T. Bodin, S. Ngo, and M. Sambridge, "The shuffling rotation of the Earth's inner core revealed by earthquake doublets," Nature Geoscience, vol. 6, no. 6, pp. 497502, 2013.

[20] P. W. Livermore, R. Hollerbach, and A. Jackson, "Electromagnetically driven westward drift and inner-core superrotation in Earth's core," Proceedings of the National Academy of Sciences of the United States of America, vol. 110, no. 40, pp. 15914-15918, 2013.

[21] G. A. Glatzmaier and P. H. Roberts, "Rotation and magnetism of Earth's inner core,” Science, vol. 274, no. 5294, pp. 1887-1891, 1996.

[22] K. Lambeck, The Earth's Variable Rotation: Geophysical Causes and Consequences, Cambridge University Press, New York, NY, USA, 1980.

[23] K. Lambeck, Geophysical Geodesy: The Slow Deformations of the Earth, Oxford University Press, New York, NY, USA, 1988.

[24] B. G. Bills, "Tidal despinning of the mantle, inner core superrotation, and outer core effective viscosity," Journal of Geophysical Research B: Solid Earth, vol. 104, no. 2, pp. 2653-2666, 1999.

[25] G. W. Wetherill, "The formation of the Earth from planetesimals," Scientific American, vol. 244, no. 6, pp. 162-174, 1981.

[26] A. H. Marcus, "Formation of the planets by accretion of planetesimals: some statistical problems," Icarus, vol. 7, no. 1-3, pp. 283-296, 1967.

[27] K. Zahnle and M.-M. M. Low, "The collision of jupiter and comet shoemaker-levy 9," Icarus, vol. 108, no. 1, pp. 1-17, 1994.

[28] G. Boué and J. Laskar, "A collisionless scenario for uranus tilting," The Astrophysical Journal Letters, vol. 712, no. 1, pp. L44L47, 2010.

[29] A. G. W. Cameron, "Cosmological considerations regarding Uranus," Icarus, vol. 24, no. 3, pp. 280-284, 1975.

[30] S. S. Sheppard, D. Jewitt, and J. Kleyna, "An ultradeep survey for irregular satellites of uranus: limits to completeness," The Astronomical Journal, vol. 129, no. 1, pp. 518-525, 2005.

[31] H. Lamb, Hydrodynamics, Dover Publications, New York, NY, USA, 1945.

[32] Z. Dai, W. Wang, and L. Wen, "Irregular topography at the Earth's inner core boundary," Proceedings of the National Academy of Sciences of the United States of America, vol. 109, no. 20, pp. 7654-7658, 2012.

[33] Ocean Tides and the Earth's Rotation, 2012, http://bowie.gsfc .nasa.gov/ggfc/tides/intro.html.

[34] J. Verhoogen, F. J. Turner, L. E. Weiss et al., The Earth, Holt Rinehart and Winston, New York, NY, USA, 1970.

[35] Y. D. Fomin, V. N. Ryzhov, and V. V. Brazhkin, "Properties of liquid iron along the melting line up to Earth-core pressures,"
Journal of Physics Condensed Matter, vol. 25, no. 28, Article ID 285104, pp. 1-5, 2013.

[36] G. A. de Wijs, G. Kresse, L. Vočadlo et al., "The viscosity of liquid iron at the physical conditions of the Earth's core," Nature, vol. 392, no. 6678, pp. 805-807, 1998.

[37] M. D. Rutter, R. A. Secco, T. Uchida et al., "Towards evaluating the viscosity of the Earth's outer core: an experimental high pressure study of liquid Fe-S (8.5 wt.\% S)," Geophysical Research Letters, vol. 29, pp. 1217-1221, 2002.

[38] S. M. Molodenskiy, "Upper viscosity boundary of the Earth's core," Iznestiya Physics of the Solid Earth, vol. 17, pp. 903-909, 1981.

[39] V. V. Brazhkin, "Investigation of the crystallization of liquid iron under pressure: extrapolation of the melt viscosity into the megabar range," Journal of Experimental and Theoretical Physics Letters, vol. 68, no. 6, pp. 502-508, 1998.

[40] V. V. Brazhkin and A. G. Lyapin, "Universal viscosity growth in metallic melts at megabar pressures: the vitreous state of the Earth's inner core," Physics-Uspekhi, vol. 43, no. 5, pp. 493-508, 2000.

[41] D. E. Smylie, "Viscosity near earth's solid inner core," Science, vol. 284, no. 5413, pp. 461-463, 1999.

[42] A. Palmer and D. E. Smylie, "VLBI observations of free core nutations and viscosity at the top of the core," Physics of the Earth and Planetary Interiors, vol. 148, no. 2-4, pp. 285-301, 2005.

[43] D. E. Smylie, V. V. Brazhkin, and A. Palmer, "Direct observations of the viscosity of Earth's outer core and extrapolation of measurements of the viscosity of liquid iron," Physics-Uspekhi, vol. 52, no. 1, pp. 79-92, 2009.

[44] D. E. Smylie, Earth Dynamics: Deformations and Oscillations of the Rotating Earth, Cambridge University Press, 2013.

[45] L. I. Lumb and K. D. Aldridge, "On viscosity estimates for the Earth's fluid outer core and core- mantle coupling," Journal of Geomagnetism \& Geoelectricity, vol. 43, no. 2, pp. 93-110, 1991.

[46] R. A. Secco, "Viscosity of the outer core," in Mineral Physics and Crystallography: A Handbook of Physical Constants, T. J. Ahrens, Ed., vol. 2, pp. 218-226, AGU, Washington, DC, USA, 1995.

[47] R. A. Muller and D. E. Morris, "Geomagnetic reversals from impacts on the Earth," Geophysical Research Letters, vol. 13, no. 11, pp. 1177-1180, 1986.

[48] G. R. Fowles and G. L. Cassiday, Analytical Mechanics, Saunders, New York, NY, USA, 6th edition, 1999.

[49] J. W. Parker, S. A. Stern, P. C. Thomas et al., "Analysis of the first disk-resolved images of ceres from ultraviolet observations with the Hubble Space Telescope," Astronomical Journal, vol. 123, no. 1, pp. 549-557, 2002.

[50] W. Alvarez, T. Rex and the Crater of Doom, Princeton University Press, Princeton, NJ, USA, 1997. 

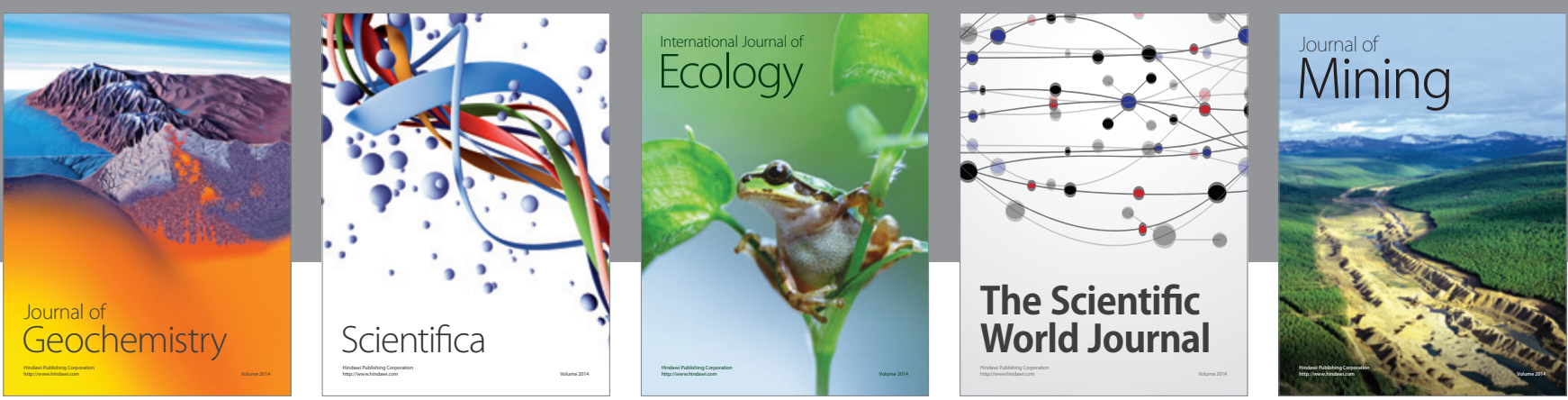

The Scientific World Journal
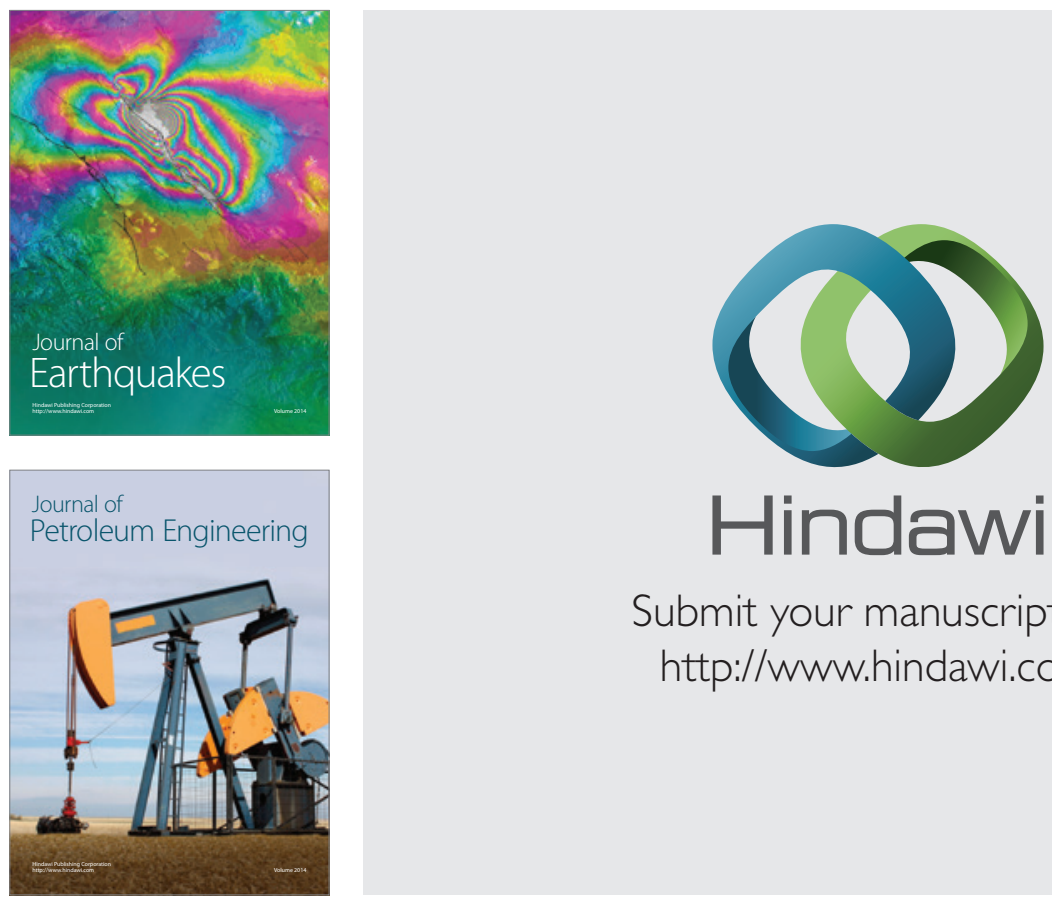

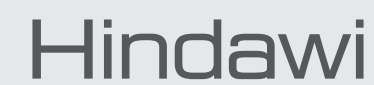

Submit your manuscripts at

http://www.hindawi.com
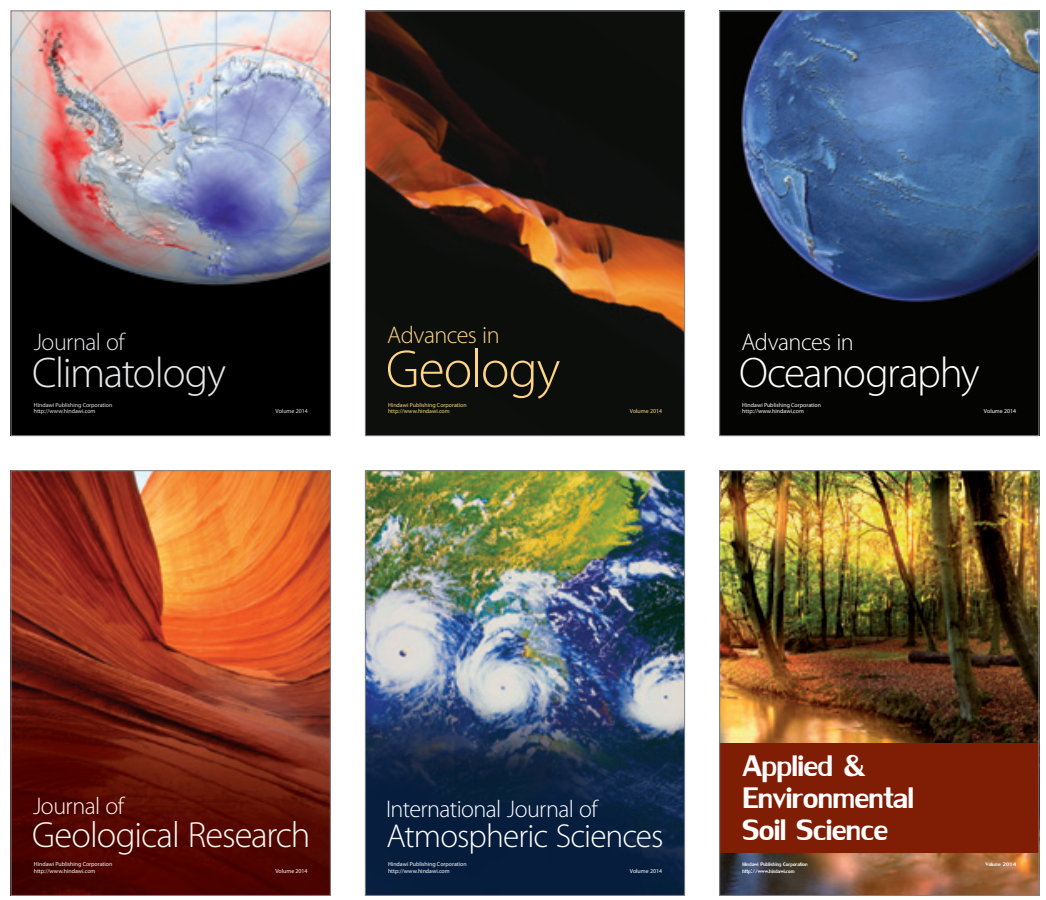
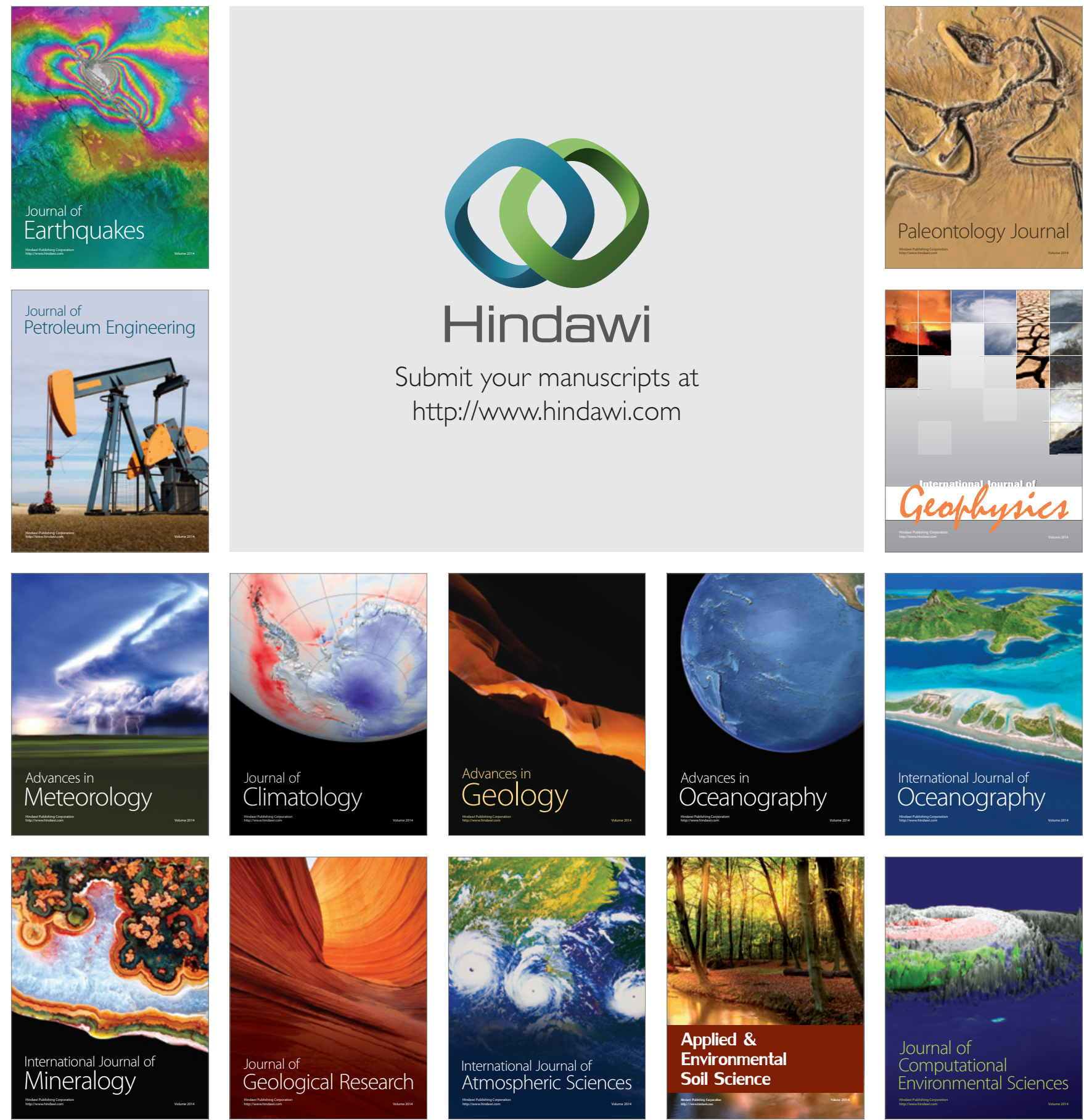